\title{
O cuidado à pessoa diabética e as repercussões na família
}

\author{
The care for the diabetic patient and family repercussion
}

La atención a la persona con diabetes y las repercusiones en la familia

\section{Maria Lúcia Zanetti', Marília Voltolini Biagg', Manoel Antônio dos Santos", Denise Siqueira Péres"II, Carla Regina de Souza Teixeira'}

'Universidade de São Paulo, Escola de Enfermagem de Ribeirão Preto. Ribeirão Preto, SP

"Universidade de São Paulo, Faculdade de Filosofia, Ciências e Letras de Ribeirão Preto. Ribeirão Preto, SP

II'Universidade de São Paulo, Faculdade de Medicina de Ribeirão Preto. Ribeirão Preto, SP

Submissão: 05/09/2007

Aprovação: 03/12/2007

\section{RESUMO}

Esse estudo teve como objetivo compreender as repercussões na família da assistência oferecida após implementação de um programa educativo em diabetes. Entrevistamos seis familiares de pacientes diabéticos de um centro universitário de Ribeirão Preto-SP, em 2007. A análise temática dos dados possibilitou identificar três temas: familiares reconhecem que o Centro constitui um diferencial para adesão ao plano alimentar, atividade física e medicamentos; o Centro é reconhecido como espaço educativo para convívio e controle do diabetes; o paciente como elemento que multiplica e expande o conhecimento acerca do diabetes na família. Os resultados apontam Que o programa educativo repercutiu favoravelmente na família, ampliando seu repertório de conhecimentos sobre o tratamento. O apoio do núcleo familiar é fundamental na adesão ao tratamento.

Descritores: Diabetes mellitus; Família; Educação em diabetes; Equipe interdisciplinar.

\section{ABSTRACT}

This study aimed to understand the repercussions in the family of the care offered after the implementation of a diabetes education program. We interviewed six relatives of diabetes patients from a university center in Ribeirão Preto-SP, Brazil, in 2007. Thematic data analysis made it possible to identify three themes: relatives recognize that the Center constitutes a differential for adherence to the food plan, physical activity and medication; the Center is acknowledged as an educative space to get together and control diabetes; the patient as an element that multiplies and expands the knowledge about diabetes in the family. The results indicate that the education program favorably affected the family, expanding its knowledge repertoire about treatment, and that the family core's support is fundamental in treatment adherence.

Descriptors: Diabetes mellitus; Family; Diabetes education; Interdisciplinary team.

\section{RESUMEN}

La finalidad de este estudio fue comprender las repercusiones en la familia de la atención ofrecida tras la implementación de un programa educativo en diabetes. Entrevistamos a seis familiares de pacientes diabéticos de un centro universitario de Ribeirão PretoSP, Brasil, en 2007. El análisis temático de los datos posibilitó identificar tres temas: familiares reconocen Que el Centro constituye un diferencial para adhesión al plano alimentario, actividad física y medicamentos; el Centro es reconocido como espacio educativo para convivencia y control del diabetes; el paciente como elemento que multiplica y expande el conocimiento acerca del diabetes en la familia. Los resultados apuntan Que el programa educativo repercutió favorablemente en la familia, ampliando su repertorio de conocimientos sobre el tratamiento, y Que el apoyo del núcleo familiar es fundamental en la adhesión al tratamiento.

Descriptores: Diabetes mellitus; Familia; Educación en diabetes; Equipo interdisciplinar. 


\section{INTRODUÇÃO}

A atenção em diabetes e às suas complicações constitui prioridade para a saúde pública mundial. Nessa direção, a prevenção em diabetes mellitus deve ser realizada nos diferentes níveis de atendimento à saúde, mediante a detecção de indivíduos em risco - prevenção primária, identificação de casos não diagnosticados prevenção secundária, e pelo tratamento dos indivíduos já afetados pela doença visando prevenir complicações agudas e crônicas prevenção terciária $^{(1)}$.

A proposta da Organização Mundial da Saúde, formulada em $2003^{(2)}$, para o atendimento de pessoas diabéticas pressupõe o envolvimento do paciente e sua família, das organizações de saúde e da comunidade no tratamento. Além disso, é fundamental o desenvolvimento de diretrizes de saúde bem definidas, formação de lideranças em saúde, planejamento de programas e locação de recursos humanos para a educação em diabetes.

É importante ressaltar a necessidade de acompanhamento, de apoio e de seguimento contínuo do paciente por uma equipe multiprofissional de saúde, pois Quando os pacientes são acompanhados de forma sistemática é possível prevenir e/ou protelar as complicações crônicas durante a evolução do Diabetes Mellitus.

O reconhecimento da importância do trabalho multiprofissional de saúde para a educação do paciente diabético propiciou o desenvolvimento de inúmeros projetos e programas educativos, tanto em nível nacional Quanto internacional ${ }^{(1,3-7)}$.

Diante do exposto, percebemos Que vários países, por meio dos profissionais, das sociedades, das instituições não governamentais e da comunidade, reconhecem a importância da reorganização do sistema de saúde para a resolução dos problemas apresentados pelos pacientes diabéticos. Nessa direção, vários projetos e programas são elaborados e implementados na tentativa de lidar com a demanda dos pacientes diabéticos, dentre eles o Staged Diabetes Management (SDM) $)^{(5)}$.

Reconhecendo a importância de prover um acompanhamento intensivo aos pacientes diabéticos, implementamos esse protocolo de atendimento no Centro Educativo de Enfermagem para Adultos e Idosos (CEEAI). Tal protocolo foi implementado em diversos países com comprovada redução nos níveis de hemoglobina glicada e diminuição das complicações advindas do diabetes ${ }^{(8)}$.

Os resultados obtidos com a implementação do SDM, no período de 2004 a 2005, no Centro Educativo de Enfermagem para Adultos e Idosos comprovou Que a sua utilização também contribuiu para melhorar o controle metabólico e a satisfação dos pacientes ali atendidos. Nesse estudo, ainda, ao investigar o apoio da família obteve-se Que 75,9\% dos pacientes relataram Que recebiam suporte familiar no início da implementação do protocolo. Esse índice aumentou para 98,1\% dos pacientes no final do programa. Esse resultado aponta Que, na percepção dos usuários, houve integração da família ao grupo de educação em diabetes no Centro Educativo em Questão $0^{(9)}$.

Nessa direção acreditamos Que ações de cuidado e educação em saúde com diabéticos devem agregar componentes da família. Desse modo, abordagem para o gerenciamento de doença crônica baseada na família pode ser considerada um acréscimo às estratégias de tratamento convencionais.

\section{A Família e a Doença Crônica}

A família pode ser considerada um contexto social nuclear no Qual os comportamentos, as ações e os hábitos de vida sofrem influência cíclica e multivetorial. Desse modo, o contexto familiar influencia fortemente o estado de saúde de cada indivíduo e este, por sua vez, influencia o modo pelo Qual a unidade familiar funciona. Nesse processo de influências recíprocas a família é considerada como o grupo primário de relacionamento e articulação entre seus membros, seja por laços biológicos, legais ou reais ${ }^{(10)}$.

Vista por esse vértice, a família representa uma unidade de cuidado ao paciente acometido por uma condição crônica de saúde. Esse cuidado é influenciado pelo meio cultural na Qual ela está inserida, pelo sistema de crenças, valores e significados compartilhados, e pelas condições sócio-econômicas e educacionais. Por essa razão, no contexto dos cuidados em saúde, a família freqüentemente toma decisões baseadas em seus hábitos de vida, crenças e valores relacionados ao processo saúde-doença, e Que sejam compatíveis com sua condição econômica ${ }^{(1)}$. O processo de tomada de decisão é mediado por esses fatores Que permeiam a relação com os serviços de saúde e Que determinam, em larga medida, a utilização dos recursos de tratamento.

Ao relacionar a doença crônica e a família, deve-se considerar a longa duração do tratamento e as conseqüentes limitações associadas ao estilo de vida cristalizado do doente e seus familiares ${ }^{(12)}$. Portanto, é fundamental compreender o processo de viver da família, Que é dinâmico e sujeito a modificações ao longo dos diferentes estágios do ciclo de vida familiar.

Em relação ao paciente diabético, o suporte familiar é fundamental, pois ele é um aliado para a aQuisição de orientações de saúde adequadas e no processo de enfrentamento da doença. Assim, o processo educativo deve valorizar as experiências e os conhecimentos antecedentes, guiado em valores e crenças do próprio paciente e de seu núcleo familiar acerca da doença ${ }^{(13)}$.

O suporte da família, como parte do contexto familiar, é apontado como significativamente associado aos comportamentos relacionados ao autocuidado no diabetes, sejam eles a dieta, o exercício ou a adesão ao tratamento medicamentoso ${ }^{(14)}$.

A família Que apóia seus membros em situação de doença compreende as modificações relacionadas à condição e torna-se permeável aos ajustamentos necessários para garantir o suporte necessário ao seu familiar doente, facilitando a adesão ao tratamento, recuperação e/ou melhora de sua saúde ${ }^{(12)}$. Desse modo podem ser asseguradas às condições para uma maior eficácia adaptativa.

Em relação ao envolvimento da família a literatura aponta ainda Que o apoio dos familiares é um requisito fundamental para que o paciente diabético consiga alcançar o automanejo de sua doença ${ }^{(15)}$.

Nesta direção, a educação em saúde aos diabéticos pode colaborar tanto para melhorar o controle metabólico da doença Quanto contribuir para Que pacientes e familiares mantenham a sua Qualidade de vida no decorrer do processo saúde-doença ${ }^{(16)}$.

Tendo em vista Que a organização familiar influencia fortemente o comportamento de saúde de seus membros e que o estado de saúde de cada indivíduo também influencia o modo como a unidade familiar funciona, infere-se Que a família é uma instituição central Que pode ajudar ou não a pessoa diabética a manejar a doença $\mathrm{e}$ alcançar as metas do seu tratamento ${ }^{(17-19)}$.

Ao considerar Que a participação familiar no processo educativo 
contribui para o seguimento do tratamento, na medida em Que serve de fonte de apoio emocional nos momentos em Que o diabético se sente impotente diante dos desafios advindos da doença, nos propomos a realizar o presente estudo.

\section{Quadro Teórico}

Para compreender a família do paciente diabético considerarse-á a Teoria dos Sistemas de Wright e Leahey ${ }^{(17)}$. Para esses autores a família é conceituada como um complexo sistema de elementos em constante interação, no Qual o cliente identifica os membros Que ele considera como família, de acordo com o significado que eles têm para seu existir.

A fundamentação dessa teoria baseia-se em cinco conceitos. O primeiro refere-se ao sistema familiar, o Qual é parte de um suprasistema mais amplo, Que por sua vez é composto de outros subsistemas. Cada supra-sistema é constituído pela comunidade e as suas interações com os grupos, as pessoas e as famílias. Os subsistemas são compostos por indivíduos Que estão inseridos nesse supra-sistema.

O segundo conceito refere-se à família como um todo, Que é diferente da soma de suas partes. Esse conceito retrata a totalidade da família como grupo, assim como suas inter-relações. O terceiro relaciona-se às interações entre os membros, nas Quais a mudança de um acaba afetando os demais integrantes da família. Esse conceito reconhece Que a interação entre os membros contribui para Que todos sofram modificações variadas Quando ocorre um fato significante com um dos componentes da família.

O equilíbrio entre a mudança e a estabilidade é o Quarto conceito, Que se refere ao estado de fluxo e alteração constantes no grupo. $\mathrm{O}$ último conceito pauta-se sobre o comportamento dos membros da família, considerando que eles devem ser compreendidos do ponto de vista de uma causalidade não-linear.

\section{IUSTIFICATIVA}

Segundo a Organização Mundial de Saúde a assistência aos indivíduos com doenças crônicas geralmente está centrada em um modelo pouco resolutivo para gerenciar as necessidades geradas por essas condições. Os modelos existentes enfocam o tratamento de doenças agudas, pautados no enfoque biomédico e fragmentado do objeto do cuidado ${ }^{(2)}$

O cuidado ao diabético, como em toda condição crônica, deve considerar a prestação de um cuidado planejado, Que envolva tempo, cenários de saúde e prestadores, treinamento para o autocuidado em domicílio, apoio dos equipamentos sociais e políticas abrangentes para o seu gerenciamento eficaz. Para efetivar esse cuidado os programas de educação em diabetes têm agregado a família como uma unidade de tratamento.

Um estudo anterior mostrou Que a família teve um papel importante para adesão do diabético ao atendimento utilizando o protocolo $S_{D M}{ }^{(5)}$. Nesta direção, acreditamos Que conhecer a visão da família acerca do atendimento oferecido aos diabéticos em um centro de extensão universitária pode fornecer subsídios importantes para o planejamento de programas de educação em diabetes. Assim, constitui como objetivo desse estudo compreender as repercussões na família do atendimento oferecido à pessoa diabética.

\section{METODOLOGIA}

Trata-se de um estudo de natureza Qualitativa. Esse tipo de estudo justifica-se porque sua abordagem é mais apropriada para responder à Questão de pesquisa: como a família percebeu o atendimento ao seu membro diabético oferecido em um centro de extensão universitária?

Os pesquisadores realizaram o estudo no Centro Educativo de Enfermagem para Adultos e Idosos (CEEAI), referido como Casa 5 pelos participantes do estudo, em janeiro de 2007. A população foi constituída pelos familiares de 54 diabéticos Que participaram de um programa de educação em diabetes. A seleção da amostra deu-se por conveniência, sendo constituída por seis familiares. Para a seleção dos sujeitos foi utilizado como critério de inclusão a freqüência de $80 \%$ a $100 \%$ às atividades programadas. Nesse estudo, consideramos família como uma rede de relacionamentos Que o paciente identifica como tal, portanto, o membro mais significativo para a pessoa diabética em suas relações familiares foi o escolhido como informante do estudo, conforme se pode observar na Tabela 1.

Tabela 1. Caracterização dos diabéticos e seus familiares, segundo idade, sexo e membro indicado pelo paciente para a entrevista. Ribeirão Preto-SP, 2007.

\begin{tabular}{ccccc}
\hline Paciente & Idade & Sexo & Membro familia & Idade \\
\hline I & 63 & Masculino & Esposa & 66 \\
2 & 60 & Feminino & Esposo & 65 \\
3 & 64 & Feminino & Filha & 40 \\
4 & 75 & Masculino & Esposa & 71 \\
5 & 71 & Feminino & Filha & 48 \\
6 & 61 & Feminino & Esposo & 62 \\
\hline
\end{tabular}

Para a coleta de dados foram utilizados: roteiro de entrevista semi-estruturado, gravador (áudio) e fitas K-7, Termo de Consentimento Livre e Esclarecido. A estrutura básica do roteiro de entrevista foi subdividida em duas partes: a primeira constou de dados de identificação pessoal dos sujeitos, e a segunda de sete Questões disparadoras.

Primeiramente realizamos contato telefônico com o membro da família indicado pelo paciente, para o Qual foram esclarecidos a natureza e os objetivos do trabalho. Uma vez tomados esses cuidados foram agendadas as entrevistas em horários prédeterminados por eles, solicitando-se Que fossem realizadas no domicílio. A entrevista foi aplicada individualmente, em situação face a face, em ambiente preservado, com condições adeQuadas de conforto. As entrevistas foram audiogravadas, tiveram duração média de 20 minutos e ocorreram após assinatura do Termo de Consentimento Livre e Esclarecido pelo paciente e respectivo familiar. O Projeto recebeu aprovação do Comitê de Ética em Pesquisa da EERP/USP em 19/06/2006, de acordo com a Resolução 196/96, protocolo no 0698/2006.

Para análise dos dados utilizou-se a análise temática de conteúdo ${ }^{(20)}$. Procedeu-se à ordenação das falas, após a transcrição integral das fitas e da leitura exaustiva do material empírico. Posteriormente selecionou-se parte do material, buscando-se as idéias relevantes Que constituem as unidades de significado, as Quais foram codificadas e organizadas pelos pesquisadores em 
subtemas, relacionando-os aos outros temas.

\section{RESULTADOS E DISCUSSÃO}

Os resultados e sua discussão serão apresentados segundo os subtemas e os temas identificados durante a análise de conteúdo temática das entrevistas.

\section{Reconhecimento do CEEAI}

Os familiares reconhecem Que o CEEAI, mediante o esforço da equipe multiprofissional, constitui um diferencial para a adesão do paciente ao plano alimentar, atividade física e medicamentos

Valoração da alimentação como recurso para o controle do diabetes

Considerando Que a restrição alimentar é uma constante no cotidiano da pessoa diabética, alcançar a meta de reeducação alimentar é um fator marcante na vida do paciente. Nessa direção, a educação em diabetes é essencial para a compreensão da necessidade de repensar o planejamento alimentar.

As falas dos familiares denotam o reconhecimento da Qualidade da alimentação como uma conseuüência do conhecimento adeuirido pelo paciente no CEEAI. A atividade educativa desenvolvida no Centro possibilitou Que o paciente compreendesse a importância e a necessidade de escolha de alimentos mais saudáveis, conforme preconizado pela pirâmide alimentar.

Mudou bastante... ela aprendeu a se alimentar melhor, a diversificar os alimentos... o valor dos alimentos... o Que ela pode ou não pode comer... (S3)

Mudanças na periodicidade com Que os pacientes se alimentavam também foram referidas pelos familiares, na medida em Que as falas mostram Que a alimentação foi fracionada de acordo com as recomendações nutricionais Que recebiam no programa educativo no CEEAI.

...começou a ver que tem que comer de três em três horas... (S I)

Nas falas abaixo se observa Que os familiares também constataram Que houve modificação na Quantidade de alimentos ingeridos, o que parece ser entendido por eles como reflexo do aprendizado adeuirido no CEEAI:

...Não deixou de comer, mas come menos... (SI)

...ela aprendeu a se alimentar melhor... e a Quantidade também...

(S3)

As falas obtidas mostram Que as famílias perceberam modificações no comportamento alimentar dos pacientes atendidos pela equipe multidisciplinar do Centro. No entanto, acredita-se Que é preciso uma rede de suporte Que envolva a família no processo de educação em diabetes. No caso particular das orientações referentes à alimentação é necessário envidar esforços para Que ocorra interação de todos os membros da família encorajando-os na busca por hábitos alimentares saudáveis. Também as orientações precisam levar em consideração os aspectos econômicos, culturais e sociais e atender as necessidades, as preocupações e os sentimentos da família acerca da Qualidade, Quantidade e fracionamento dos alimentos.

\section{Suporte e apoio na terapia medicamentosa}

O conhecimento acerca dos medicamentos utilizados para controle do diabetes foi apontado pelo familiar como um ganho no tratamento. Mediante o conhecimento adQuirido sobre o medicamento utilizado é possível compreender e relacionar mecanismos de ação, dosagem, horário e efeitos colaterais.

...além de ela Questionar bastante o médico, ela se interava através da própria bula e através de outros Questionamentos... Sabe praticamente tudo, da reação de cada medicamento, da finalidade de cada um, ela está bem por dentro. (S6)

O cumprimento do horário prescrito dos medicamentos é um ponto de dificuldade no tratamento de QualQuer doença, pois acarreta mudança nos hábitos e reorganização do cotidiano do paciente, principalmente em terapia medicamentosa de uso contínuo, como no caso das do diabetes. A educação em diabetes auxilia o paciente no cumprimento dos horários à medida Que ele compreende a importância dos mecanismos de ação e efeitos colaterais.

Nas falas abaixo os familiares evidenciam Que perceberam mudanças no horário ou no cumprimento dos horários dos medicamentos que seu familiar diabético usa, e se mostram capazes, também, de compreender a sua importância. Isso denota Que a educação em diabetes não ficou restrita somente para o paciente atendido no CEEAI. A família também passou a ser conhecedora das orientações ali fornecidas e assim pôde exercer mais pontualmente seu papel de cuidadora, o Que mostra o efeito multiplicador do aprendizado.

...ele tomava depois do almoço; aí depois, lá na Casa, foi explicado Que podia tomar uma hora antes. Então foi uma mudança... (SI) ...ficou mais consciente, toma os medicamentos no horário certo... (S3)

Outras constatações identificadas pelos familiares referem-se aos ajustes terapêuticos realizados Quando da sua participação no programa educativo do CEEAI:

\section{...Depois que ele começou a ir na Casa 5 ele começou a usar insulina... (SI) \\ ...Só fracionou, metade cedo, metade à tarde. (S4)}

As falas acima denotam que o conhecimento acerca da terapia medicamentosa não ficou restrito ao paciente, mas tem repercussão no contexto da família. Os familiares demonstram ter conhecimento dos medicamentos utilizados pelo diabético Quanto ao nome, horário, bem como a introdução de outros medicamentos.

\section{Inclusão da prática da atividade física no tratamento e mudança nos hábitos do familiar diabético}

A prática de atividade física envolve mudança de hábitos 
pessoais, alteração no cotidiano, motivação, força de vontade, valores, crenças e auto-estima, sendo, portanto, de difícil adesão. Iniciar e/ou incrementar a atividade física é um dos objetivos do tratamento dispensado ao paciente diabético, com o apoio da equipe multiprofissional. As falas abaixo relacionadas referem-se às modificações ocorridas nos hábitos de vida referente à prática de atividade física pelos pacientes.

\section{...Ela não praticava esporte nenhum. Hoje ela vai fielmente na hidroginástica, não falta a nenhuma aula... (S3)}

Os familiares notaram não só a incorporação da prática de atividade física no cotidiano, como parte do tratamento, como também uma mudança na organização dos hábitos e no cotidiano de seu familiar diabético.

Pacientes Que recebem apoio familiar são aqueles Que mais conhecem sua enfermidade, aderem ao tratamento medicamentoso e ao plano alimentar, bem como incrementam a atividade física; conseqüentemente, apresentam melhor controle metabólico ${ }^{(15)}$.

\section{O CEEAI é reconhecido como espaço educativo para o convívio e controle do diabetes}

Os familiares relacionam o trabalho desenvolvido pela equipe com a melhora significativa do paciente na aceitação $e$ enfrentamento da doença

O diagnóstico de diabetes tem um impacto profundo no paciente. Lidar com um tratamento contínuo e com as complicações desencadeadas pelo controle metabólico insatisfatório é muito difícil para o diabético. O sentimento de impotência e revolta dificulta o enfrentamento e o convívio com as demandas diárias Que a doença impõe (21). Assim, o apoio psicológico, o suporte e os esclarecimentos oferecidos por todos os profissionais da equipe são identificados como essenciais para fortalecer os pacientes a desenvolverem estratégias de enfrentamento desde o estabelecimento do diagnóstico do paciente.

As falas abaixo revelam a importância do trabalho multiprofissional desenvolvido no espaço do CEEAI:

Porque lá ... eles ensinam, lá eles fazem ver que a diabetes não é coisa do outro mundo. É uma coisa que você pode conviver, pode comer, pode beber, pode andar, pode fazer esportes, pode fazer tudo. Desde que você saiba lidar com ela ... Então a pessoa fica consciente. Ele melhorou bem depois que ele começou a ir lá... a diabete dele estabilizou, a pressão... eu acho que ele se conformou, estabilizou, sabe como agir. Então pra ele foi muito bom. (SI)

...melhorou a consciência dele, porQue no começo ele teve muita dificuldade em aceitar... na Casa 5 ele passou a aceitar muito bem a diabetes, muito bem. (S4)

Os depoimentos revelam Que a equipe multiprofissional constitui uma referência para os pacientes atendidos e conta com o reconhecimento dos seus familiares Quando esses referem Que houve uma melhora significativa na aceitação e enfrentamento da doença por parte do diabético. Estudos demonstram Que as redes de apoio psicossocial auxiliam as famílias a desenvolverem meios para superação das dificuldades, minimizando a sobrecarga cotidiana resultante do convívio com uma pessoa com condição crônica na família(22;23).

Por outro lado, reconhece-se Que as atividades desenvolvidas precisam explorar certos aspectos psicológicos e estratégias educativas, pois ainda há pacientes Que apresentam baixa adesão ao plano alimentar.

...não come na hora, sempre fora de hora... já teve duas vezes hipoglicemia... (S4)

Nessa fala a dificuldade do paciente em estabelecer horários para alimentação é bem evidenciada por seu familiar, capaz de relacionar tal dificuldade com os episódios de hipoglicemia.

A interferência da família na terapêutica é justificada pela alteração infligida na estrutura familiar Quando um dos membros sofre com algum tipo de problema. A família tende a buscar soluções e opinar na tentativa de resolver o problema de seu familiar da melhor forma.

...eu acho que ele come muita fruta. Minhas filhas já falaram para ele que não é pra ele comer tanta fruta... (S4)

Desmistificação do tratamento com insulina como evidência de gravidade da doença

O trabalho desenvolvido pela equipe multiprofissional colaborou para a superação do medo e da resistência frente à introdução da insulina para o tratamento do diabetes, conforme sugerem as falas abaixo relacionadas.

Quando disse que era bom ele tomar a insulina, houve medo... a gente da família ficou meio assim. Mas depois ele viu Que a insulina pra ele tava fazendo melhor. (SI)

...o grande trauma do tratamento... foi o uso da insulina... Evidente Que a Casa 5, com as orientações recebidas, ela assimilou de uma forma melhor. Eos profissionais da Casa 5 foram um grande apoio nesse sentido... usando hoje como uma rotina normal. (S6)

A indicação da insulina no tratamento parece ser um momento de apreensão para o paciente e sua família. A utilização da insulina pode assumir um significado de materialização da gravidade da doença, suscitando o medo de lidar com o desconhecido. Perceberse transitando nas fronteiras do desconhecido pode trazer ansiedade não apenas ao paciente, como aos seus familiares. Apesar da apreensão inicial vivenciada ao se introduzir a insulina no tratamento, a superação dos pacientes no presente estudo, segundo os seus familiares, deveu-se ao apoio fornecido pela equipe multiprofissional do CEEAI.

\section{O paciente como elemento Que expande o conhecimento acerca do diabetes na família}

O paciente assumindo também o papel de cuidador no ambiente familiar

Algumas falas possibilitam perceber como o conhecimento adouirido acerca do diabetes e de seu tratamento pôde ser expandido para o núcleo familiar. 
...ela estendeu isso pra família inteira, tanto em casa como para outros filhos e outros netos... Olha, geralmente mesmo depois das reuniões, a gente discute muito assim, o que foi falado lá dentro. Ela tenta passar isso para todos os familiares, tanto os Que estão na casa residindo, como para o resto da família Que vem, um amigo... Então tudo isso, ela passou a ser assim mais reguladora com os membros da família... (S5)

...doce aqui havia em profusão, foi o primeiro corte... ela foi aplicando os ensinamentos, as orientações Que ela recebeu na Casa 5. (S6)

Desse modo, percebe-se Que o projeto educativo repercutiu para além dos limites do CEEAI, ou seja, transcendeu o âmbito estritamente pessoal do paciente, atingindo também o núcleo familiar, onde os pacientes passaram a exercer também o papel de cuidadores, disseminando informações, levando o conhecimento para ajudar os membros da família na aQuisição de hábitos saudáveis Que contribuem para a prevenção do diabetes mellitus entre pessoas sadias. As teorias de aprendizagem evidenciam que a possibilidade de ensinar aquilo que se aprendeu é uma forma eficaz de consolidar o aprendizado. Observa-se, assim, Que o trabalho multidisciplinar pôde chegar às famílias e transformar hábitos e práticas instituídos.

A informação é primordial para os diabéticos, sobretudo Quando instrumentaliza a família ${ }^{(23)}$. Porém é válido dizer Que, tomada isoladamente, a informação é de pouca valia. O manuseio da informação é importante Quando potencializa o triângulo terapêutico equipe multidisicplinar, família e equipamentos sociais. Torna-se relevante na medida em Que fornece apoio para mudança de comportamento, aumentando a autonomia do paciente e minimizando os problemas encontrados no processo de enfrentamento da doença.

A compreensão da importância de proceder aos ajustes terapêuticos para o controle do diabetes foi percebida pelos familiares, pois o tratamento de doenças crônicas reQuer ajustes na terapêutica ao longo do tempo para Que se tenha um controle satisfatório. Quando o paciente e sua família estão bem orientados Quanto a essa necessidade, a aceitação e colaboração com o tratamento acontecem de forma menos traumática.

...se ela tiver Que vir a fazer uso, ela não vai ser resistente não.

Ela acha que o que for melhor pra sobrevida dela e pra Qualidade de vida... (S5)

A fala acima denota Que o familiar demonstra ter confiança nas orientações da equipe multiprofissional, pois caso haja necessidade de introdução de novos fármacos a expectativa de Que haja aceitação por parte da paciente é bastante positiva, antecipando o enfrentamento esperado pela filha da paciente, no caso de ela vir a usar insulina. Em contraposição a esses resultados, um outro estudo demonstrou Que famílias Que enfrentam uma condição crônica apresentam necessidades e expectativas Que nem sempre são atendidas em seus contatos com os profissionais de saúde ${ }^{(24)}$.

Por outro lado, observam-se também avanços obtidos Quanto ao conhecimento acerca da auto-aplicação da insulina, conforme depoimento abaixo.

...Depois que ele começou a ir na Casa 5, ele começou a usar insulina. Então ele sabe a validade, Quando tem que trocar, Que tem Que ficar na geladeira, Quando viaja, a dosagem certa, Quando viaja tem Que pôr em caixa de isopor. Ele se programou. Ele estudou. Ele perguntava tudo direitinho. Como até hoje ele põe tudo certinho. (SI)

A família refere Que a aQuisição de conhecimento pelo paciente durante a sua participação no CEEAI foi intensa e que ele atualmente conhece o processo de administração da insulina, compartilhando esse conhecimento para a toda a família.

\section{CONSIDERAÇÕES FINAIS}

Os dados obtidos nessa investigação mostraram Que o programa educativo oferecido no Centro por equipe multidisciplinar teve repercussão na dinâmica da família, ampliando o conhecimento de seus membros em relação ao tratamento e controle do diabetes. As falas obtidas possibilitaram compreender Que o paciente constituiu um elemento Que expande o conhecimento acerca do diabetes na família e esta passou a exercer mais adequadamente seu papel de cuidadora. A ampliação do aprendizado pode favorecer a aQuisição de hábitos saudáveis na família. Desse modo, compreendemos Que a educação em diabetes deve estar centrada na equipe multidisciplinar, no sistema familiar, no paciente e nos equipamentos sociais. Quando o paciente encontra essa rede de apoio há uma maior efetividade no processo educativo.

\section{REFERÊNCIAS}

I. Ministério da Saúde (BR), Organização Pan-Americana da Saúde. Avaliação do plano de reorganização da atenção à hipertensão arterial e ao diabetes mellitus no Brasil. Brasília (DF): Ministério da Saúde; 2004.

2. Organização Mundial da Saúde. Cuidados inovadores para condições crônicas: componentes estruturais de ação: relatório mundial. Brasília (DF): Organização Mundial da Saúde; 2003.

3. Organización Panamericana de la Salud. Atlas de educación en diabetes en América Latina y el Caribe: inventario de programas para personas con diabetes tipo 2. Washington (DC): Organización Panamericana de la Salud; 2002.

4. García R, Suárez R, Mateo de Acosta O. Programa de educación en diabetes del Instituto Nacional de Endocrinologia: una estratégia de comunicación interactive. Rev Asoc Latinoam Diabetes 1993; 3: 112-5.

5. Mazze R, Weaver T, Upham P, Simonson G, Bradley R, Sundem $S$, et al. Staged Diabetes Management - Decision Support (SDM-ds): an internet-based system for clinical decision making. Diabetes Res Clin Practice 2000; 50 (suppl.): 196.

6. Grupo de Estudio de la Diabetes en la Atención Primaria de Salud (GEDAPS). Guía para el tratamiento de la diabetes tipo 2 en la Atención Primaria: contribuición al programa de acción de la declaración de St. Vicent. $3^{\text {a }}$ ed. Madrid (ESP): Ediciones 
Harcourt SA; 2000.

7. Ministério da Saúde (BR). Plano de reorganização da atenção à hipertensão arterial e ao diabetes mellitus. Brasília (DF): Ministério da Saúde; 2002.

8. Mazze RS, Simonson GD, Strok E, Bergenstal R, Idrogo M, Ramirez S, et al. Staged Diabetes Management: a systematic evidence-based approach to the prevention and treatment of diabetes and its co-morbidities. Pract Diabetes Int 200 I; 18(7): SI-SI6.

9. Otero LM. Atendimento ao paciente diabético utilizando o protocolo Staged Diabetes Management [tese]. Ribeirão Preto (SP): Escola de Enfermagem de Ribeirão Preto, Universidade de São Paulo; 2005.

10. Potter PA, Perry AG. Cuidado nas famílias. In: Potter PA, Perry AG, organizadores. Fundamentos de Enfermagem. $5^{\text {a }}$ ed. Rio de Janeiro (RI): Guanabara Kogan; 2004. p. I 17-29.

I1. Althof CR, Elsen I, Laurindo AC. Família: o foco de cuidado na Enfermagem. Texto Contexto Enferm 1998; 7(2): 320-7.

12. Moreira TM, Araújo TL, Pagliuca LMF. Alcance da teoria de King junto à família de pessoas portadoras de Hipertensão Arterial Sistêmica. Rev Gaúcha Enferm 200 I ; 22(2): 74-89.

13. Santos ECB, Zanetti ML, Otero LM, Santos MA. O cuidado sob a ótica do paciente diabético e seu principal cuidador. Rev Latino-am Enfermagem 2005; 13(3): 397-406.

14. Albright TL, Parchman M, Burge SK. Predictors of self-care behavior in adults with type 2 diabetes: an RRNeST study. Fam Med 200 I ; 33(5): 354-60.

15. Rodríguez-Morán M, Guerrero-Romero JF. Importancia del apoyo familiar en el control de la glicemia. Salud Pública Méx 1997; 39(1): 44-7.
16. Cazarini RP, Zanetti ML, Ribeiro KP, Pace AE, Foss MC. Adesão a um grupo educativo de pessoas portadoras de diabetes mellitus: porcentagem e causas. Med Ribeirão Preto 2002; 35(2): 142-50.

17. Wright LM, Leahey M. Enfermeiras e famílias: um guia para avaliação e intervenção na família. $3^{a}$ ed. São Paulo (SP): Roca; 2002.

18. Armour TA. The effctiveness of family interventions in people with diabetes mellitus: a systematic review. Diabete Med 2005; 22(10): 1464-591.

19. Ferraz AEP, Zanetti ML, Brandão ECM, Romeu LC, Foss MC, Paccola GMGF, et al. Atendimento multiprofissional ao paciente com diabetes mellitus no Ambulatório de Diabetes do Hospital das Clínicas da Faculdade de Medicina de Ribeirão Preto. Medicina 2000; 33(2): 1 70-5.

20. Minayo MCS. Pesquisa social: teoria, método e criatividade. Petrópolis (RI): Vozes; 1996.

21. Péres DS. Sentimentos, pensamentos e comportamentos de mulheres portadoras de diabetes tipo 2 [dissertação]. Ribeirão Preto (SP): Faculdade de medicina de Ribeirão Preto, Universidade de São Paulo; 2004.

22. Pettengil MAM, Angelo M. O sentido de cuidar da criança e da família na comunidade: a experiência da aluna de enfermagem. Rev Esc Enferm USP 2000; 34(I): 91-8.

23. Nunes MDR, Dupas G, Ferreira NMLA. Diabetes na infância/ adolescência: conhecendo a dinâmica familiar. Rev Eletrônica Enferm 2007; 9 (1): 119-30.

24. Elsie I, Marcon SS, Silva MRS. O viver em família e sua interface com a doença. Maringá (PR): Editora Universidade Estadual de Maringá; 2002. 